\title{
Conectivas anormales y vaguedad
}

\section{(1) Paula Teijeiro}

Instituto de Investigaciones Filosóficas-Sociedad Argentina de Análisis Filosófico -Consejo Nacional de Investigaciones Científicas y Técnicas- Universidad de Buenos Aires, Argentina

Recibido el 20/1/2018; aceptado el 5/3/2018.

\section{Resumen}

En el presente artículo defiendo la existencia de conectivas lógicas vagas. Para ello, expongo primero sus antecedentes históricos y conceptuales en términos de la teoría de cuantificadores generalizados. Luego, propongo una definición de vaguedad específica para ellas, y muestro el vínculo entre el fenómeno predicativo y el operacional.

\section{Abnormal connectives and vagueness}

\begin{abstract}
In this paper, I defend the existence of vague logical connectives. In order to sustain this claim, I first present some historical and conceptual precedent of these ideas, in terms of the theory of generalized quantifiers. I then propose a definition of vagueness specific to them, and show the link between the predicative and the operational phenomenon. ${ }^{1}$
\end{abstract}

\section{Introducción}

Los predicados vagos son aquellos con áreas grises de aplicación, que dan lugar a paradojas de Sorites (ver por ejemplo Gómez Torrente, 2014, Teijeiro, 2015). La inmensa mayoría de la literatura acerca de la vaguedad se aboca justamente al fenómeno tal como se manifiesta en predicados, mientras que una proporción notablemente menor se ocupa de los nombres. La posibilidad de que haya conectivas lógicas vagas no sólo es rechazada de modo universal en los pocos lugares donde es considerada, sino que ello se hace sin siquiera un análisis superficial de la cuestión. Hasta el momento, la posibilidad de vocabulario lógico vago permanece fundamentalmente inexplorada.

En el presente trabajo pretendo remediar este olvido, defendiendo que hay operadores lógicos vagos. A pesar de lo extraño que pueda parecer en el ámbito filosófico la

\section{Palabras clave}

Anormalidad conectivas lógicas cuantificadores vaguedad

\section{Keywords}

Abnormality logical connectives quantifiers vagueness

1. Quisiera agradecer especialmente a los referis anónimos de este artículo por sus aportes y correcciones, y por la celeridad de su trabajo. 
idea de una conectiva vaga, dentro de la lingüística hace más de medio siglo que se consideran cuantificadores que poseen casos limítrofes de aplicación. En la sección II voy a hacer un breve repaso por los antecedentes históricos que desembocaron en la teoría de los cuantificadores generalizados. En la sección III voy a ofrecer una taxonomía muy general de estos operadores, y mostrar en qué sentido algunos de ellos son vagos. Veremos que el motivo es que ellos también pueden entenderse como predicados, en particular predicados de segundo nivel. Lo que pretendo mostrar es que la posibilidad de conectivas vagas no sólo no es una idea tan disruptiva como parece, sino que al igual que la vaguedad cuantificacional, también puede entenderse en continuidad natural con las de los predicados. En la sección IV, finalmente, voy a argumentar que la vaguedad de conectivas corresponde con la propiedad de anormalidad, que intuitivamente consiste en poder arrojar outputs indeterminados al recibir inputs precisos.

Este artículo no asume demasiados conocimientos de lógica, aunque sí ciertas nociones básicas. Por otro lado, toda la discusión se encuentra enfocada desde una perspectiva modelo-teórica, puesto que considerar otras teorías de la vaguedad nos apartaría demasiado del punto, además de introducir complicaciones técnicas sustantivas. Por último, si bien motivado por fenómenos presentes en el lenguaje natural, la investigación acerca de conectivas lógicas vagas no es en principio el análisis de un fenómeno necesariamente existente en él, sino más bien una exploración de un campo conceptual, por el momento, relegado a lenguajes formales.

\section{Breve historia de la cuantificación}

Si bien la contribución de los cuantificadores a la forma lógica es el aporte más fundamental del silogismo aristotélico al desarrollo del análisis estructural de los argumentos, no es hasta fines del siglo XIX que se logra una comprensión de ellos independiente de las categorías gramaticales. La inspiración para el surgimiento de la lógica simbólica proviene fundamentalmente de distintas ramas de la matemática; en lo relativo en particular a estos operadores podemos identificar dos grandes aportes a la concepción contemporánea, provistos por los desarrollos -independientes- de dos filósofos matemáticos.

Por un lado tenemos a Peirce, quien se inscribe en la tradición algebraica del giro matemático en lógica, y que comienza por elaborar la distinción entre cuantificadores y operadores boooleanos. En (1885) ya encontramos una concepción de ellos como abreviaturas de fórmulas infinitas; en particular, versiones infinitarias de las operaciones aritméticas de suma y producto.

En segundo lugar está la Conceptografía (1879) de Frege. Allí, el objetivo es ofrecer un lenguaje formal universal y riguroso, carente de las desprolijidades propias de los lenguajes naturales, y proveerlo de una teoría de la prueba que permita reducir todo argumento correcto a una serie de pasos mecánicos. La construcción de este lenguaje se basa en una ontología constituida por dos tipos de entidades: las saturadas, que son los objetos, y las no saturadas, que son los conceptos. Dado que las expresiones lingüísticas deben referir a alguna entidad para ser significativas, nos encontramos con que los predicados refieren a conceptos y los nombres, a objetos.

Dentro de las dos posibilidades que ofrece la nueva ontología, los cuantificadores sólo pueden referir a conceptos. Sin embargo, sus objetos de aplicación no son simplemente entidades saturadas, sino que son a su vez otros conceptos. En Los fundamentos de la aritmética (1884) Frege llama a los cuantificadores conceptos de segundo nivel, retomando el enfoque aristotélico que había sido abandonado en pos del análisis en 
términos de sujeto y predicado, y reemplazándolo por la concepción contemporánea de funciones y argumentos.

Ya entrado el siglo XX, las ideas de Frege se abren en un campo que hoy se conoce como Teoría de los cuantificadores generalizados. El primero en proveer un aparato formal y probar resultados para cuantificadores distintos de "todos" y "alguno" es Mostowski (1957). Su proyecto consiste en caracterizar a los cuantificadores como operadores que ligan variables, y que semánticamente son interpretados como funciones que van de la potencia del dominio en $\{0,1\}$. A partir de ello, define toda la familia de "exactamente $n$ ", pero también otros que no son definibles en lógica de primer orden, como "hay una finita cantidad de cosas en el dominio" o "más de la mitad de las cosas del dominio".

Algunos años más tarde Rescher (1962) retoma las ideas de Mostowski, pero su interés se desplaza de la mera generalización matemática de operaciones, hacia la interpretación de expresiones del lenguaje natural. En particular, Rescher se pregunta cómo modelar el cuantificador "la mayoría" (que hoy en día se conoce en la literatura como cuantificador Rescher), y ofrece ciertos principios -aunque no una axiomatización completa- que lo regulan. También en Montague (1973) encontramos la idea de interpretar a los cuantificadores del lenguaje natural como clases de clases, aunque su interés va más allá del cuantificador de Rescher.

Finalmente, Zadeh (1983) -inventor de la teoría de conjuntos difusa- da el siguiente paso y enmarca a los cuantificadores como un capítulo del proyecto de desarrollar una lógica que sirva como herramienta para la inteligencia artificial. Esto abre la puerta a considerar aún más cuantificadores, ya no sólo aquellos que resultan indefinibles en primer orden, sino también los que aparentan escapar a las reglas de la lógica clásica en general.

\section{Teoría de los cuantificadores generalizados}

Como dijimos, la teoría de los cuantificadores generalizados parte de comprender a $\forall$ y $\exists$ en primer lugar como un tipo particular de conceptos. Dado un modelo formado por un conjunto de objetos $\mathrm{D}$ que constituye su dominio de discurso, los cuantificadores tradicionales pueden definirse como:

Definición (Cuantificador preciso) Un cuantificador preciso es una función de subconjuntos del dominio en $\{0,1\}$. $^{2}$

Los predicados, que también significan conceptos, se interpretan mediante conjuntos de objetos del dominio. Esos conjuntos quedan determinados por lo que se llama su función característica, que va del dominio a los valores o y 1. Del mismo modo, los cuantificadores pueden considerarse como significando conceptos de segundo nivel porque sus interpretaciones son las funciones características de conjuntos que toman como argumentos conjuntos de objetos en lugar de simplemente objetos.

Sin embargo, no toda propiedad de segundo nivel es un cuantificador, sino que se trata en particular de propiedades numéricas, como "tener la misma cardinalidad que el dominio del modelo", en el caso de $\forall$, o "no ser vacío", en el caso de $\exists$ :

$$
\begin{aligned}
\underline{\exists} \Psi=\begin{array}{l}
1 \text { si } \operatorname{card}(\Psi) \neq \varnothing \\
0 \text { en otro caso }
\end{array} \quad \underline{\forall} \Psi=1 \text { si } \operatorname{card}(\Psi)=\operatorname{card}(\mathrm{D}) \\
0 \text { en otro caso }
\end{aligned}
$$

2. Estrictamente, las definiciones de los cuantificadores deberían generalizarse a n-tuplas en lugar de ser simplemente subconjuntos del dominio, de modo tal de permitir cuantificadores de aridad mayor 1. Esto puede parecer innecesario para el lógico poco interesado en el lenguaje natural, dado que oraciones como "todos los $A$ son $B^{\prime \prime}$ pueden formalizarse apelando a un cuantificador unario "todos", y un condicional que restrinja su alcance. Sin embargo, este procedimiento no puede emplearse para otros casos, como "más de la mitad de los $A$ son $B^{\prime \prime}$. A modo de ejemplo, la primera oración no puede parafrasearse como la segunda:

(3) Más de la mitad de los gatos son blancos.

(4) Más de la mitad de las cosas, o no son gatos, o son blancos.

Barwise \& Cooper (1981) son quienes prueban en general esta imposibilidad de reducción. Por ende, estos cuantificadores restringidos son operaciones irreductiblemente diádicas:

half $(\Phi, \Psi)=1$ si $\operatorname{card}(\Phi \cap \Psi)>\operatorname{card}(\Phi) / 2$ Desde el punto de vista sintáctico, esto puede representarse de muchas maneras. Para evitar introducir variables de segundo orden, lo más sencillo es agregar al lenguaje de primer orden cuantificadores que ligan variables objetuales al igual que $\forall y \exists$, pero que funcionan sintácticamente como operadores n-arios. Por ejemplo, la formalización de (3) sería: half $x((G x)(B x))$ que debe leerse como "la mitad de las cosas que son gatos, son blancos", sin que ese "que" pue da reducirse a un condicional. 
Donde $\operatorname{card}(\Psi)$ refiere a la cardinalidad de $\Psi$ y, en general, usaremos la notación $\underline{X}$ para significar el valor semántico de X. Ejemplos de otras operaciones además de $\exists$ y $\forall$-no necesariamente definibles en primer orden- que son cuantificadores precisos en este sentido son:

$$
\begin{aligned}
& \exists^{3} \Psi=\quad 1 \text { si } \operatorname{card}(\Psi)=3 \quad \underline{\exists^{* 0}} \Psi=1 \text { si } \operatorname{card}(\Psi)=\aleph_{0} \\
& 0 \text { en otro caso } 0 \text { en otro caso } \\
& \forall^{1 / 2} \Psi=\quad 1 \mathrm{si} \operatorname{card}(\Psi)=\operatorname{card}(\mathrm{D}) / 2 \quad \underline{\forall^{-k}} \Psi=1 \mathrm{si} \operatorname{card}(\Psi)=\operatorname{card}(\mathrm{D})-k \\
& 0 \text { en otro caso } 0 \text { en otro caso }
\end{aligned}
$$

El segundo paso en la expansión de la teoría cuantificacional consiste en notar que esas mismas operaciones a veces se aplican en el lenguaje natural a argumentos vagos:

(1) Todos son altos.

Un modo de representar formalmente esos argumentos es como conjuntos difusos, esto es, conjuntos cuyas funciones características tienen como codominio el intervalo [0;1]. Ahora bien, la operación correspondiente a "todo" que definimos más arriba, cuando se aplica a un argumento difuso como "altos", puede resultar demasiado estricta. Por ejemplo, si todas las personas en cuestión tienen una altura mayor que el promedio, aunque quizás no todas sea determinadamente altas, (1) resultaría tan falsa como si alguno de ellos fuera sin dudas petiso.

La lógica difusa es una teoría que permite lidiar con oraciones que contengan predicados referentes a esos conjuntos difusos. Para ello, también se deben reinterpretar los cuantificadores, de modo que típicamente, cuando los argumentos sean precisos, den los resultados clásicos, y cuando no lo sean, den resultados más tolerantes:

Definición (Cuantificador difuso) Un cuantificador difuso es una función de subconjuntos posiblemente difusos del dominio en $[0,1]$.

Todo cuantificador preciso es difuso, aunque no a la inversa. Para modelar los cuantificadores "todos" y "alguno" del lenguaje natural, las funciones que usualmente se utilizan son las propuestas por la lógica difusa de Łukasiewicz, presentada por primera vez en (1920):

$$
\underline{\exists}^{t} \Psi=\operatorname{supr}(\Psi) \quad \underline{\forall^{\star}} \Psi=\inf (\Psi)
$$

donde supr y inf son operaciones que arrojan respectivamente el supremo y el ínfimo valor de la función característica del conjunto.

Ahora bien, existe otro fenómeno de indeterminación que se refleja en oraciones como

\section{(2) Muchos son altos}

3. Los cuantificadores del lenguaje natural presentan más habitualmente aridad 2 que otras aridades; pero es particularmente extraño pensar en cuantificadores vagos de aridad 1 . El cuantificador de (2) puede entenderse como restringido "a la fuerza" por el dominio, pero sin dudas no podremos hacer eso si queremos interpretar a la vez diversas oraciones. Dado que en última instancia nuestro interés final son las conectivas, no nos detendremos en este problema. También estamos obviando, en pos de simplificar la presentación, todos los matices que provienen del hecho de que estos cuantificadores son sin dudas relativos al contexto.
Aquí no se trata de generalizar la operación clásica, es decir, de dar mejores interpretaciones para los mismos cuantificadores. Por el contrario, "muchos" es un cuantificador nuevo y la operación que lo representa es difusa incluso para conjuntos clásicos. Para distinguirlos dentro del caso general de cuantificadores difusos, llamaremos a estos vagos o aproximados, y a los difusos que no son vagos, (como "todos", de acuerdo con la lectura de Łukasiewicz), exactos:

Definición (Cuantificador vago) Un cuantificador vago es un cuantificador difuso que ofrece valores intermedios para algunos de sus argumentos precisos. ${ }^{3}$ 
Definición (Cuantificador exacto) Un cuantificador exacto es un cuantificador difuso que ofrece valores clásicos para todos sus argumentos precisos.

Vamos a usar el símbolo $\forall$ como representación formal de un cuantificador vago genérico, como "muchos", "bastantes", etc. Ofrecer una representación veritativo funcional de los cuantificadores difusos más allá de "todos" y "alguno" no es una tarea fácil. El motivo de ello es que se trata de propiedades determinadas por la cardinalidad de los conjuntos, y no hay una única manera de contar cosas para el caso de conjuntos difusos. Si en una habitación hay solo cinco personas altas y 5 personas bajas, podemos fácilmente decir que la mitad de las personas de la habitación son altas. Pero si hay tres personas altas, tres personas bajas, y cuatro personas que son un caso limítrofe de "alto" ¿debemos contarlas como altas o como no-altas? ¿o contabilizarlas de algún modo intermedio?

Esta cuestión no es peculiar de la vaguedad de los cuantificadores sino de la de sus argumentos. Y dado que justamente la de los primeros puede ocurrir incluso en ausencia de la de los segundos, podemos avanzar postergando este problema, y asumiendo que cuando hablemos de la cardinalidad de un conjunto -aun difuso-estamos refiriéndonos a un concepto adecuadamente definido.

Una ventaja de igualar la vaguedad de los cuantificadores a la de los predicados es que no resulta ser un fenómeno misterioso, radicalmente distinto, o siquiera meramente emparentado al tradicional: es exactamente el mismo. Por ende, todo lo que sabemos de unos puede trasladarse a los otros. En particular, vamos a considerar aquí cómo se manifiestan para los cuantificadores dos de los atributos fundamentales de los predicados vagos: los casos limítrofes y la sensibilidad a Sorites (en esta línea, Solt (2011) ofrece una comparación entre las palabras de la clase de many y los adjetivos graduados).

Respecto de los casos limítrofes, para los conceptos vagos de primer nivel, como "pelado", podemos identificarlos semánticamente porque la función característica del predicado le asigna algún valor distinto de 1 y o. Los conceptos de segundo nivel son distintos en ese sentido, del hecho de que $\forall A$ tenga un valor intermedio no se puede inferir que $\underline{A}$ sea un caso limítrofe de $\forall$, ya que el valor indeterminado podría provenir de la vaguedad de $A$. No tener esto en cuenta nos llevaría a considerar a los cuantificadores exactos como poseedores de casos limítrofes, dado que $\forall A$ puede recibir valores intermedios. Por el contrario, la vaguedad de los cuantificadores es independiente de la de sus argumentos: si tenemos un conjunto con cien mil personas sin un solo pelo, aunque haya también una persona de calvicie incipiente, el predicado "muchos" se aplica sin dudas a ese conjunto.

Intuitivamente, un caso limítrofe de un cuantificador vago es una cantidad que no sea ni determinadamente muchos objetos (sea $k$ ), ni determinadamente no-muchos (sea $m$ ). Todo conjunto con una cantidad $n$ de elementos tal que $m<n<k$ va a ser un caso limítrofe de $\forall$. Necesariamente, algunos de esos $\Psi$ limítrofes serán conjuntos precisos, dado que todos los subconjuntos de objetos del dominio de discurso están en el dominio de la función $\forall$. Ellos son los que de hecho evidencian la vaguedad del predicado, aunque también habrá conjuntos difusos que sean limítrofes.

Veamos entonces en segundo lugar cómo se manifiesta el fenómeno sorítico para los cuantificadores. La paradoja de Sorites es el argumento que permite derivar un absurdo a partir de la premisa de que los predicados vagos son tolerantes, es decir, que pequeños cambios no son suficientes para alterar el valor de verdad de las oraciones que los involucran: 
(10.3.

\footnotetext{
4. En primer lugar, dado que no toda conectiva es conmutativa, estrictamente los argumentos no deberían ser simplemente conjuntos de oraciones, sino -al igual que con los cuantificadores- ser secuencias. En segundo lugar, no toda caracterización de una conectiva será una medida simple de cantidad, así como no todo cuantificador generalizado lo es. Por ejemplo, podríamos tener una conectiva de aridad 10 que expresara "más de las primeras cinco que de las segundas cinco oraciones son verdaderas" -lo cual sería equivalente a un cuantificador proporcional.
}

Un ser humano de 30 años es una persona

Si algo es una persona, un instante antes también lo era.

Por ende, un embrión es una persona.

Respecto de "muchos", un ejemplo (similar al) que ofrece Solt misma es:

1000 estudiantes son muchos.

Si n estudiantes son muchos, n-1 también son muchos.

Por ende, 0 estudiantes son muchos.

Este argumento, formalizado utilizando nuestro lenguaje de primer orden con el cuantificador vago unario (suponiendo que el dominio está compuesto sólo de estudiantes) sería:

$$
\begin{aligned}
& \operatorname{card}(\Sigma)=1000 \rightarrow \forall x E x \\
& \forall n((\operatorname{card}(\Sigma)=n \rightarrow \forall x E x) \rightarrow(\operatorname{card}(\Sigma)=n-1 \rightarrow \forall x E x)) \\
& \hline \operatorname{card}(\Sigma)=0 \rightarrow \forall x E x
\end{aligned}
$$

Donde $\Sigma$ es el nombre del conjunto de estudiantes y $E x$ es el predicado "ser un estudiante". El rol de la relación de semejanza para predicados lo cumple aquí la cardinalidad de los conjuntos a los que se aplica $\forall$. Vale la pena notar que la paradoja no descansa en la vaguedad de $E x$, que es prima facie un predicado suficientemente preciso.

Por ende, hemos visto hasta aquí cómo, a pesar de ser ignorados casi por completo en la literatura filosófica, los cuantificadores vagos son un fenómeno lingüísticamente estudiado, y perfectamente análogo al de los predicados tradicionales. Veremos en la próxima sección entonces cómo esto podría trasladarse al caso ya sí inexplorado de las conectivas lógicas.

\section{Conectivas lógicas}

El punto de partida es que las conectivas también pueden pensarse como predicados de cantidad, pero a diferencia de los cuantificadores, sus argumentos no son conjuntos de objetos, sino de oraciones. ${ }^{4}$ La cantidad entra en juego en tanto que afirman que algún número de sus argumentos es verdadero: la negación afirma que ninguno lo es, la disyunción, que lo es al menos uno; la conjunción, que son los dos, y así. Si $\tau_{v}(\Psi)$ es el subconjunto de $\Psi$ que son oraciones verdaderas en $v$, entonces:

$$
\begin{aligned}
& v\left(\operatorname{conj}\left({ }^{r} A^{\top},{ }^{r} B^{\top}\right)\right)=1 \text { si } \operatorname{card}\left(t_{v}\{A, B\}\right)=2 \\
& 0 \text { en otro caso } \\
& \mathrm{v}\left(\operatorname{disy}\left({ }^{\circ} \mathrm{A}^{\top},{ }^{\prime} \mathrm{B}^{\top}{ }^{\top}\right)\right)=1 \operatorname{si} \operatorname{card}\left(\mathrm{t}_{\mathrm{v}}\{\mathrm{A}, \mathrm{B}\}\right) \geq 1 \\
& 0 \text { en otro caso }
\end{aligned}
$$

De ese modo, si podemos ver a las conectivas como números, podemos pensar en la posibilidad de un número difuso. Y para que sean cantidades vagas, al igual que los cuantificadores, deben poder tener casos indeterminados, incluso si sus argumentos son precisos. Esta propiedad es conocida en la literatura como Anormalidad, y su vínculo con la vaguedad no ha sido establecido que yo sepa hasta el momento.

La normalidad es una propiedad de sistemas proposicionales no-clásicos, raramente mencionada, dada su ubicuidad: casi todas las conectivas son normales. Rescher (1968) atribuye las primeras investigaciones a Emil Post (1921) y Alonzo Church, en 
un texto inhallable, publicado en el boletín de la sociedad mexicana de matemática en (1953), llamado Non-Normal Truth-Tables for the Propositional Calculus:

Diremos que es normal la tabla de verdad para una conectiva proposicional que sea el análogo multivaluado de una de las conectivas bivalentes si incluye por lo menos un valor análogo a la verdad $\mathrm{T}$ (...) y por lo menos un valor análogo a la falsedad $\mathrm{F}(. .$.$) , y$ esta tabla multivaluada acuerda por completo con la tabla estándar bivalente para la conectiva en C cuando sólo los valores T y F están involucrados. (Rescher, 1968: 78).

Priest, por su parte, la define del siguiente modo:

Llámese normal a una lógica multivaluada en el lenguaje del cálculo proposicional clásico si entre sus valores hay dos, 1 y 0, tales que 1 es designado, 0 no lo es, y para toda función veritativa correspondiente a una conectiva, el output para esos inputs es el mismo que el output clásico. (Priest, 2008: 140).

La idea general es que se trata de conectivas que serían clásicas, de no existir los fenómenos que precipitan la adopción de valores de verdad extraordinarios. No debe confundirse esto con otro concepto emparentado, que se encuentra en Carnap (1942). Allí, lo que puede o no ser normal son la valuaciones, y no las conectivas, y una valuación es anormal cuando no respeta las restricciones booleanas.

Un poco más precisamente, la caracterización de la propiedad que nos interesa es la siguiente:

Definición (Normalidad Intrínseca): Una conectiva $\odot$ es normal sii hay alguna tabla que resulta de eliminar de la tabla de $\odot$ las filas y columnas de todos los valores menos uno designado* -reetiquetado como 1- y uno no designado* reetiquetado como 0 - y en el interior de esa tabla sólo aparecen 1 s y 0s.

Un raro ejemplo de conectiva anormal presente en la literatura es el condicional de la lógica de Cantwell (2008), que no recibe valor 1 cuando su antecedente recibe valor o: ${ }^{5}$

\begin{tabular}{c|ccc}
\multicolumn{1}{l}{$\rightarrow$} & 1 & $1 / 2$ & 0 \\
\cline { 2 - 4 } 1 & 1 & $1 / 2$ & 0 \\
$1 / 2$ & 1 & $1 / 2$ & 0 \\
0 & $1 / 2$ & $1 / 2$ & $1 / 2$
\end{tabular}

La aclaración de que se trata de una propiedad intrínseca consiste en que pedimos que la restricción coincida con alguna operación booleana. Rescher, cuando habla de "el análogo multivaluado de una conectiva bivalente", nos da la pauta de que aquello en lo que está pensando es una relación entre conectivas de un lenguaje-clásico- y conectivas de otro -no clásico. Sin embargo, este concepto relacional de normalidad no nos sirve, dado que nosotras queremos evaluar conectivas que no son siquiera definibles en un lenguaje clásico.

Veamos entonces en qué consisten los casos limítrofes y las paradojas soríticas formuladas con operaciones proposicionales. En primer lugar, los casos limítrofes no pueden ser simplemente cualquier conjunto que dé como resultado un valor intermedio, puesto que eso haría vaga a casi cualquier conectiva de una lógica no clásica. Por el otro lado, tampoco pueden ser casos limítrofes sólo aquellos conjuntos que den valores intermedios pero sólo contengan oraciones clásicas. 
Las conectivas vagas afirman que una cantidad difusa de sus argumentos -"muchos"son verdaderos. Por ende, los casos limítrofes serán aquellos conjuntos cuya cantidad de argumentos verdaderos no sea ni muchos ni no-muchos. En base a eso, podemos definir el predicado con que representa alguna conectiva vaga de aridad $n$ por ejemplo así:

$$
\begin{aligned}
\left(\operatorname{con}\left({ }^{\top} A_{1}, \ldots,{ }^{\prime} A_{\mathrm{n}}\right)\right)= & 1 \text { si } \operatorname{card}\left(\mathrm{t}_{\mathrm{v}}\left\{A_{1}, \ldots, A_{\mathrm{n}}\right\}\right)>n \\
& 0 \text { si } \operatorname{card}\left(\mathrm{t}_{\mathrm{v}}\left\{A_{1}, \ldots, A_{\mathrm{n}}\right\}\right)<n \\
& 1 / 2 \text { en otro caso }
\end{aligned}
$$

De este modo, todo conjunto que tenga $n$ oraciones verdaderas será un caso limítrofe de con.

Respecto de Sorites, la forma de la paradoja resulta muy semejante a la de los cuantificadores. Hay sólo quizás una salvedad que debemos aclarar, que es cuantitativa pero significativa. El rango de aplicación de un predicado está en general conformado por una gran cantidad de objetos, lo cual hace que la pendiente resbaladiza sea "suave". Por el contrario, las conectivas usuales en general tienen aridad baja, con lo cual la secuencia sorítica no estará constituida de demasiados pasos.

Esto no significa que no podamos construir secuencias soríticas con conectivas, sino que en muchos casos el principio de inducción no tendrá tanto peso. Sorites más y menos atractivos también pueden construirse con predicados: la secuencia para "ser un montón de arena" es más difícil de detener que la secuencia para el ya mencionado "ser una religión".

\section{Conclusión}

En este artículo he motivado la idea de que no hay razones para excluir ciertas categorías linguísticas de la posibilidad de comportarse vagamente. Esto es importante básicamente por dos cuestiones, una de cariz más filosófico, y otra más formal.

En primer lugar, la incorporación de la vaguedad a la lógica -como algo distinto de la modificación de la lógica para lidiar con una vaguedad que no le es propia- es la última frontera de la indeterminación. Si logramos cruzarla, tendremos un buen argumento en favor del antiexcepcionalismo, esto es, la idea de que las teorías lógicas forman un continuo con otras teorías, tanto formales como empíricas.

En segundo lugar, las lógicas anormales constituyen una familia de sistemas que ha recibido mucha menor atención en el campo de la investigación no clásica, justamente por el hecho de que lo que se busca es estudiar extensiones de operaciones precisas. La preocupación por conectivas vagas abre así un campo teórico relativamente novedoso.

Por último, queda un guante tendido que debe ser levantado por las ciencias empíricas, que es la pregunta por si el fenómeno está de hecho presente en el lenguaje natural, o se trata de una posibilidad conceptual o formal, sin correlato en ninguna lengua existente. 


\section{Bibliografía}

" Barwise, J., y R. Cooper (1981). Generalized Quantifiers and Natural Language. Linguistics and Philosophy, 4, 159-219, doi: 10.1007/BF00350139.

" Cantwell, J. (2008). The Logic of Conditional Negation. Notre Dame Journal of Formal Logic, 49 (3), 245-260, doi: 10.1215/00294527-2008-010

»Carnap, R. (1942). Introduction to Semantics, Studies in Semantics, vol. 1. Cambridge: Harvard University Press.

" Church, A. (1953). Non-normal Truth-tables for the Propositional Calculus. Boletín de la Sociedad Matemática Mexicana, 10, 41-52, doi: 10.2307/2268648.

»Frege, G. (1967). Concept Script, a formal language of pure thought modelled upon that of arithmetic [Begriffsschrift, eine der arithmetischen nachgebildete Formelsprache des reinen Denkens (1879)]. (trad. S. Bauer-Mengelberg). En J. van Heijenoort (Ed.). From Frege to Gödel: A Source Book in Mathematical Logic, 18791931. Cambridge: MA: Harvard University Press,.

» Frege, G. (1974). The Foundations of Arithmetic: A logico-mathematical enquiry into the concept of number [Die Grundlagen der Arithmetik: eine logisch mathematische Untersuchung über den Begriff der Zahl (1884)]. (Trad. J.L. Austin) (2da ed.). Oxford: Blackwell.

» Gómez Torrente (2014). La paradoja Sorites. En Barrio, E. (Ed.) Paradojas, paradojas y más paradojas. London: College Publications.

» Łukasiewicz, J., (1920). O logice trojwartosciowej. Ruch Filozoficny, 5: 170-171. Traducido al inglés en Borkowski, L. (Ed.). (1970). Selected Works. Amsterdam: North-Holland and Warsaw: PWN.

" Montague, R. (1973). The proper treatment of quantification in ordinary English. En Kulas J., Fetzer J.H., Rankin T.L. (Eds). Philosophy, Language, and Artificial Intelligence. Studies in Cognitive Systems, vol 2. Dordrecht: Springer, doi: 10.1007/97894-009-2727-8_7.

» Mostowski, A. (1957). On a generalization of quantifiers. Fundamenta mathematicae, 44, 12-36, doi: 10.2307/2964414.

"Peirce, C.S. (1885). On the Algebra of Logic. A Contribution to the Philosophy of Notation. American Journal of Mathematics, 7, 197-202, doi: 10.2307/2369451.

» Post, E. (1921). Introduction to a General Theory of Elementary Propositions. American Journal of Mathematics, 43, 163-185.

»Priest, G. (2008). An introduction to non-classical logics, Cambridge: Cambridge University Press, doi: 10.1017/CBO9780511801174.

» Rescher, N. (1962). Plurality Quantification. Reproducido en (2004) Plurality Quantification Revisited. Philosophical Inquiry, 26 (1-2). 1-6, doi: 10.5840/philinquiry2004261/21.

"Solt, S. (2011). Vagueness in quantity: Two case studies from a linguistic perspective. En Cintula, P.. Fermueller, C. y Godo, L. (Eds.). Understanding vagueness. logical, philosophical and linguistic perspectives. London: College Publications.

"Teijeiro, P. (2015). Lógicas no clásicas de la vaguedad. Revista de Humanidades de Valparaíso, 5, 7 -16, doi: 10.22370/rhv.2015.5.135. 
"Zadeh, L.A. (1983). A computational approach to fuzzy quantifiers in natural languages. Computers and Mathematics with Applications, 9 (1), 149-184. doi: 10.1016/0898-1221(83)90013-5. 